\title{
Inflammatory/immune-suppressive microenvironment characteristics of pancreatic cancer patients with Shi-Re ZHENG identified by extracellular vesicle long RNA profiling
}

\section{Shulin Yu}

Fudan University Shanghai Cancer Center

\section{Yawen Geng}

Fudan University Shanghai Cancer Center

\section{Ling Qian}

Fudan University Shanghai Cancer Center

\section{Kun Chen}

Fudan University Shanghai Cancer Center

\section{Yalei Zhang}

Fudan University Shanghai Cancer Center

\section{Shenglin Huang}

Fudan University Shanghai Cancer Center

Ye Li

Fudan University Shanghai Cancer Center

Peng Wang ( $\sim$ peng_wang@fudan.edu.cn)

Fudan University Shanghai Cancer Center https://orcid.org/0000-0003-0569-9001

\section{Research}

Keywords: Pancreatic cancer, inflammatory/immune microenvironment, Shi-Re ZHENG, extracellular vesicle long RNA

Posted Date: May 4th, 2021

DOl: https://doi.org/10.21203/rs.3.rs-474915/v1

License: (9) (1) This work is licensed under a Creative Commons Attribution 4.0 International License. Read Full License 


\section{Abstract \\ Background}

Extracellular vesicles (EVs) contain long RNAs that reflect their tissue origins and the relative fractions of different immune cell types. This study examined the molecular basis of different traditional Chinese Medicine ZHENG diagnoses (also called syndrome differentiation) in pancreatic ductal adenocarcinoma (PDAC).

\section{Methods}

A total of 128 PDAC patients with different ZHENG diagnoses were retrospectively reviewed in this study. The EV long RNA profile of plasma samples was analyzed by long RNA sequencing. Differentially regulated EV long RNAs were annotated and assessed for Gene Ontology pathway enrichment using DAVID. Cell-type enrichment analysis was performed using the online program $x$ Cell.

\section{Results}

Approximately 15000 annotated genes, mainly including mRNAs, were reliably detected in each sample. Different ZHENG diagnoses exhibited unique EV mRNA expression profiles and therefore different enriched pathways. Gene Set Enrichment Analysis identified TGF- $\beta$ and KRAS signaling activation as the hallmarks of cancer with Shi-Re ZHENG. Cell-type enrichment analysis also revealed a varied inflammation/immune cell type distribution among patients with or without Shi-Re diagnosis. Mast cells, platelets and Tregs were significantly enriched but basophils, CLP, DC, and CDC cells were decreased in patients with Shi-Re diagnosis compared with patients without Shi-Re diagnosis.

\section{Conclusions}

We identified the hallmarks of cancer with different ZHENG diagnoses based on plasma EV long RNA sequencing. In particular, TGF- $\beta$ and KRAS signaling activation were the hallmarks of Shi-Re ZHENG, which contribute to shape an inflammatory/immune-suppressive tumor microenvironment in PDAC.

\section{Background}

Traditional Chinese Medicine (TCM) has a unique theoretical system for the treatment of cancers and has accumulated abundant clinical experience for a prolonged period [1]. TCM emphasizes the overall coordination of the environment inside and outside the human body [2] and focuses attention on the individual rather than the disease. ZHENG, also called the TCM syndrome, is the core of clinical TCM and vital for the modernization of TCM. ZHENG is based on comprehensive analysis of clinical information collected through the main TCM diagnosis procedures: observation, listening, questioning, and pulse 
analyses. ZHENG differentiation is the most essential principle that guides the prescription of Chinese herbal formulas [3].

With the rapid development of sequencing analysis and bioinformatics, studies have used a combination of genomics, transcriptomics, proteomics, metabolomics and data analysis to examine the molecular basis of TCM ZHENG [4-10]. Screening, identification and verification of characteristic, objective markers of ZHENG have also become critical in the research of TCM ZHENG $[11,12]$

Extracellular vesicles (EVs), which are released by cells, are made up of a membrane structure with a lipid bilayer that contains various functional contents, including proteins, lipids, and RNA [13-16]. EVs stably and effectively mediate intercellular communication by loading and transporting proteins and nuclear acids $[17,18]$. We previously developed an optimized strategy for plasma EV long RNA sequencing. More than 10000 exLRs, including mRNAs, circRNAs, and IncRNAs, were reliably detected in each EV long RNAs -seq sample from 1-2 mL of plasma [19]. We found that blood EV long RNAs reflected their tissue origins and the relative fractions of different immune cell types [20]. Additionally, the EV long RNA profile could distinguish patients with cancer from healthy individuals [21]. Therefore, EV long RNAs may help identify diverse specific markers potentially useful for cancer diagnosis and help study the molecular basis of TCM ZHENG.

In this study, we analyzed the EV mRNA expression in different ZHENG types among patients with pancreatic ductal adenocarcinoma (PDAC). We examined the enrichment pathways and cell component variations of the different ZHENG of PDAC patients based on plasma EV long RNA sequencing.

\section{Methods}

\section{Patients and clinical characteristics}

This retrospective cohort study was conducted in 128 patients who had been pathologically diagnosed with PDAC from November 2018 to September 2019. According to the International Union Against Cancer TNM Classification of Malignant Tumors (eighth edition), locally advanced pancreatic cancer (stage III) was defined as tumor invasion of the celiac trunk, the superior mesenteric artery, or both. TNM stage was determined by imaging diagnosis using techniques such as magnetic resonance imaging or computed tomography.

The study was approved by the Fudan University Shanghai Cancer Center Ethics Committee (Ethical No.: 050432-4-1212B). The methods used in this study were performed according to the ethical standards of our committee and the tenants of the 1964 Declaration of Helsinki and its later amendments. Written informed consent was obtained from each patient according to the committee's regulations.

\section{Collection of plasma samples}

Plasma samples were collected from all participants in 10-mL EDTA-coated Vacutainer tubes as previously reported [21]. Plasma samples were collected at least three days prior to initiating major 
therapy. Plasma was separated by centrifugation at $800 \times g$ for $10 \mathrm{~min}$ at room temperature $\left(25^{\circ} \mathrm{C}\right)$ within $2 \mathrm{~h}$ after blood collection and centrifuged at $16,000 \times \mathrm{g}$ for $10 \mathrm{~min}$ at $4^{\circ} \mathrm{C}$ to remove debris. Plasma samples were stored at $-80^{\circ} \mathrm{C}$ until use.

\section{Isolation of EVs and EV long RNA}

The isolation of EVs and EV long RNA were performed as previously reported [21]. For each patient, $1 \mathrm{~mL}$ of plasma was used, and EVs were isolated by affinity-based binding to spin columns using an exoRNeasy Serum/Plasma Kit (Qiagen, Hilden, Germany) following the manufacturer's instructions. Briefly, thawed plasma was mixed with binding buffer and added to the exoEasy membrane-affinity spin column. For transmission electron microscopy (TEM), size distribution measurement, and western blotting, the EVs were eluted with $400 \mu \mathrm{L}$ of XE elution buffer. To reduce the eluate volume (to $50 \mu \mathrm{L}$ ) and exchange buffer with PBS, samples were subjected to ultrafiltration using the Amicon Ultra- 0.5 Centrifugal Filter 10kDa (Merck Millipore, Germany). For EV RNA isolation, EVs were lysed on the column using QIAzol (Qiagen), and total RNA was then eluted and purified.

\section{TEM}

TEM was performed as previously reported [21]. Briefly, EVs were identified by negative staining with phosphotungstic acid. Fifty microliters of resuspended EVs were placed on a Parafilm membrane. A copper mesh with a formvar supporting membrane was covered with the EV suspension and floated for 3-10 min to allow sample absorption into the supporting membrane. Next, $50 \mu \mathrm{L}$ of $2 \%$ phosphotungstic acid was dropped onto the Parafilm membrane. The fluid was then absorbed from the edges of the copper mesh with filter paper. The copper mesh absorbing the sample was covered with $2 \%$ phosphotungstic acid and floated for $3 \mathrm{~min}$. The sample was then dried for $10 \mathrm{~min}$ under incandescent light after the staining solution was absorbed with filter paper. The copper mesh was imaged with a transmission electron microscope (Phillips CM120, Tokyo, Japan).

\section{Size distribution measurement}

Size distribution analysis of EVs was performed with a Flow NanoAnalyzer (NanoFCM Inc., Xiamen, China) according to the manufacturer's instructions, as previously reported [21]. A series of monodispersed silica nanoparticles were synthesized and used as size reference standards. The side scattering (SSC) distribution histogram of the mixture was obtained. The SSC intensity of every vesicle was converted into its corresponding vesicle size. The EV samples resuspended in $100 \mathrm{~mL}$ of phosphatebuffered saline (PBS) and $100 \mathrm{~mL}$ of PBS as a blank control were analyzed using the same instrument settings. The EV data were analyzed and used to construct a size distribution histogram.

\section{Western blotting}

Western blotting was performed as previously reported [21]. Peripheral blood mononuclear cells (PBMCs) and concentrated EVs were lysed in RIPA buffer [ $1 \%$ NP40, $0.5 \%$ deoxycholate, $0.1 \%$ sodium dodecyl sulfate (SDS) in Tris-buffered saline] with complete protease inhibitors. Equal amounts of proteins were 
separated by $10 \%$ SDS-polyacrylamide gel electrophoresis and transferred to a nitrocellulose membrane (Bio-Rad, Hercules, CA, USA). Membranes were blocked with $5 \%$ non-fat milk and incubated with primary antibodies. Target proteins were detected using an enhanced chemiluminescence kit (Amersham Pharmacia Biotech, Uppsala, Sweden). The following primary antibodies were used: anti-GRP94 (147001-AP, 1:1,000), anti-calnexin (10427-2-AP, 1:1,000) (Proteintech, Rosemont, IL, USA), anti-CD63 (ab92726, 1:1,000) (Abcam, Cambridge, USA), and anti-TSG101 (sc-13611, 1:500) (Santa Cruz Biotechnology, Dallas, TX, USA).

\section{RNA sequencing (RNA-seq) analysis}

RNA-seq analyses were performed as previously reported [19-21]. Total EV RNA isolated from $1 \mathrm{~mL}$ of plasma was treated with DNase I (NEB, Ipswich, MA, USA) to remove DNA. Strand-specific RNA-seq libraries were prepared using the SMARTer ${ }^{\circledR}$ Stranded Total RNA-Seq Kit - Pico Input Mammalian (Clontech, Palo Alto, CA, USA). Library quality was analyzed using a Qubit fluorometer (Thermo Fisher Scientific, Waltham, MA, USA) and Qsep100 (BiOptic, New Taipei City, Taiwan). EV RNA-seq libraries were prepared from $1 \mathrm{~mL}$ of plasma on average. RNA-seq was performed on an Illumina sequencing platform (San Diego, CA, USA) with 150-bp paired-end run metrics.

Raw reads were filtered using FastQC and aligned to the GRCh38 human genome assembly using HISAT2. Gene expression levels were calculated in transcripts per kilobase million (TPM). Annotations of mRNA in the human genome were retrieved from the GENCODE (v25). Differentially regulated exLRs were annotated gene IDs and assessed for Gene Ontology (GO) pathway enrichment using DAVID (https://david.ncifcrf.gov/). Cell type enrichment analysis of genes was performed using xCell (https://xcell.ucsf.edu).

\section{Statistical analyses}

Student's t-test was used for the statistical analysis. Statistical analyses were two sided and a $P$-value $<$ 0.05 was considered statistically significant. Statistical analyses were performed using SPSS software (SPSS v.21), GraphPad Prism (v6.0, La Jolla, CA, USA), and R software (v3.4.2).

\section{Results}

\section{Patient characteristics}

A total of 190 patients with PDAC were enrolled in the study. Among the 190 patients, 65 patients (34.2\%) were diagnosed as Shi-Re ZHENG, 44 patients (23.2\%) were Pin-Xu ZHENG, 30 patients (15.8\%) were XueYu ZHENG, 27 patients (14.2\%) were Yin-Xu ZHENG, and 24 patients (12.6\%) were non-TCM syndrome (NS). Patients whose syndrome were insidious and could not be classified as a specific ZHENG were be classified as NS, according to a previous report [22]. Plasma was available for 128 patients, and these samples were then subjected to further EV mRNA sequencing. The demographic and clinical characteristics of the 128 patients are shown in Table 1. 
Table 1

Clinical characteristics of PDAC patients with different ZHENG diagnoses.

\begin{tabular}{|c|c|c|c|c|c|c|}
\hline \multicolumn{2}{|l|}{ Variables Value } & \multirow{2}{*}{$\begin{array}{l}P i-X u \\
(n=34) \\
21\end{array}$} & \multirow{2}{*}{$\begin{array}{l}X u e-Y u \\
(n=25)\end{array}$} & \multirow{2}{*}{$\begin{array}{l}\text { Shi-Re } \\
(n=23)\end{array}$} & \multirow{2}{*}{$\begin{array}{l}\text { Yin-Xu } \\
(n=22) \\
18\end{array}$} & \multirow{2}{*}{$\begin{array}{l}N S \\
(n=24) \\
9\end{array}$} \\
\hline Age, (years) & $\geq 60$ & & & & & \\
\hline & $<60$ & 13 & 6 & 6 & 4 & 15 \\
\hline \multirow[t]{2}{*}{ Sex } & Male & 20 & 19 & 15 & 11 & 19 \\
\hline & Female & 14 & 6 & 8 & 11 & 5 \\
\hline \multirow[t]{2}{*}{ Tumor stage } & III & 8 & 8 & 6 & 9 & 4 \\
\hline & IV & 26 & 17 & 17 & 13 & 20 \\
\hline \multirow[t]{2}{*}{ Tumor location } & Head/neck & 18 & 17 & 11 & 14 & 10 \\
\hline & Body/tail & 16 & 8 & 12 & 8 & 14 \\
\hline CA19-9 & $\geq 1000$ & 12 & 9 & 12 & 9 & 3 \\
\hline$(\mathrm{IU} / \mathrm{ml})$ & $<1000$ & 22 & 16 & 11 & 13 & 21 \\
\hline \multirow[t]{2}{*}{ Metastasis } & Intrahepatic Extrahepatic & 16 & 11 & 10 & 7 & 12 \\
\hline & & 10 & 6 & 7 & 6 & 8 \\
\hline
\end{tabular}

\section{Plasma EV mRNA sequencing results}

Plasma EVs were obtained as described in Material and Methods. TEM analysis showed the presence of rounded, cup-shaped, double-membrane-bound vesicle-like structures, typical of EVs (Fig. 1a). The size distribution measurements showed that the isolated vesicles were heterogeneous populations with an average diameter of $79.5 \mathrm{~nm}$ (Fig. 1b). Western blot analysis of plasma samples from the PDAC patients confirmed the successful extraction of EVs by revealing the presence in isolated vesicles of CD63, TSG101 (Fig. 1c), which were not observed for any of the antibodies in PBMCs. Grp94 and calnexin were observed in PBMCs but not in the isolated vesicles (Fig. 1c), demonstrating the efficiency of EV extraction.

ExLR-seq was then performed using plasma samples from the 128 PDAC patients with different ZHENG diagnoses. Approximately 15,000 annotated genes, including mRNAs, IncRNAs and pseudogenes, were reliably detected in each sample. Numbers of detected RNA species did not significantly differ between groups (Fig. 1d).

\section{Hallmarks of cancer among patients with different TCM ZHENG diagnosis identified by EV mRNA sequencing}


Unsupervised hierarchical clustering was first used to analyze the EV mRNA expression among samples from patients with different ZHENG diagnoses. As shown in Fig. 2a, patients with different ZHENG diagnoses exhibited unique EV mRNA expression patterns. A clear separation of patients with Shi-Re ZHENG from patients with Non-Shi-Re ZHENG based on the heatmap was also noticed. Pathway analysis revealed that differentially expressed EV mRNAs were enriched for some pathways among different ZHENG diagnoses. As shown in Fig. 2b, platelet degranulation, inflammatory response and angiogenesis pathway were enriched among patients with Pi-Xu. Transcription, DNA-templated, cellular response to interferon-gamma, and the cell chemotaxis pathway were enriched among patients with Xue-Yu. Fibrinolysis, cell differentiation, cell surface receptor signaling pathway, and angiogenesis were enriched among patients with Yin-Xu. Other pathways, including cellular response to interleukin-1, cellular response to interferon-gamma, cell adhesion, extracellular matrix organization, and cellular response to tumor necrosis factor, were enriched among patients with Shi-Re. GSEA was conducted to further analyze the hallmarks gene sets $(n=50)$ that represent the specific and definite biological processes among different ZHENGs. Comparison among different ZHENGs was performed and the results are shown in Table 2. We identified the hallmarks of cancer among different ZHENG diagnoses, such as TNF-a signaling in Yin-Xu and apical-surface in Xue-Yu. We also identified TGF- $\beta$ and KRAS signaling activation as the hallmarks of cancer with Shi-Re ZHENG. 
Table 2

Hallmark gene sets in PDAC patients with different ZHENG diagnoses.

\section{$P$-value}

\begin{tabular}{|c|c|c|c|c|c|}
\hline Hallmarks $(n=50)$ & $\begin{array}{l}\text { Pi-Xu } \\
\text { vs. NS }\end{array}$ & $\begin{array}{l}\text { Yin-Xu } \\
\text { Vs. NS }\end{array}$ & $\begin{array}{l}\text { Xue-Yu } \\
\text { vs. NS }\end{array}$ & $\begin{array}{l}\text { Shi-Re } \\
\text { vs. NS }\end{array}$ & $\begin{array}{l}\text { Shi-Re vs. } \\
\text { Non-Shi-Re }\end{array}$ \\
\hline TNFA_SIGNALING_VIA_NFKB & 0.9301 & 0.0289 & 0.1144 & 0.4828 & 0.0868 \\
\hline HYPOXIA & 0.3190 & 0.5688 & 0.7178 & 0.1641 & 0.1518 \\
\hline CHOLESTEROL_HOMEOSTASIS & 0.7762 & 0.8950 & 0.5571 & 0.1848 & 0.1483 \\
\hline MITOTIC_SPINDLE & 0.2050 & 0.8936 & 0.7895 & 0.1274 & 0.1144 \\
\hline WNT_BETA_CATENIN_SIGNALING & 0.2986 & 0.3087 & 0.3008 & 0.4614 & 0.0812 \\
\hline TGF_BETA_SIGNALING & 0.1826 & 0.6145 & 0.6045 & 0.0317 & 0.0345 \\
\hline IL6_JAK_STAT3_SIGNALING & 0.8949 & 0.0810 & 0.0859 & 0.7215 & 0.2178 \\
\hline DNA_REPAIR & 0.4118 & 0.0495 & 0.2913 & 0.1813 & 0.0557 \\
\hline G2M_CHECKPOINT & 0.7760 & 0.7377 & 0.7963 & 0.0766 & 0.0669 \\
\hline APOPTOSIS & 0.5769 & 0.0487 & 0.6263 & 0.6662 & 0.8633 \\
\hline NOTCH_SIGNALING & 0.3878 & 0.0599 & 0.0658 & 0.4176 & 0.7055 \\
\hline ADIPOGENESIS & 0.0722 & 0.3609 & 0.3983 & 0.1262 & 0.2009 \\
\hline ESTROGEN_RESPONSE_EARLY & 0.1112 & 0.6341 & 0.6110 & 0.1194 & 0.2544 \\
\hline ESTROGEN_RESPONSE_LATE & 0.0017 & 0.5607 & 0.8587 & 0.0008 & 0.0317 \\
\hline RESPONSE & 0.0900 & 0.5100 & 0.7407 & 0.8052 & 0.6838 \\
\hline MYOGENESIS & 0.4831 & 0.5492 & 0.3085 & 0.0560 & 0.0490 \\
\hline PROTEIN_SECRETION & 0.8971 & 0.5911 & 0.9923 & 0.1362 & 0.0816 \\
\hline INTERFERON_ALPHA_RESPONSE & 0.0409 & 0.7418 & 0.3719 & 0.0836 & 0.1919 \\
\hline INTERFERON_GAMMA_RESPONSE & 0.1238 & 0.5690 & 0.7707 & 0.0626 & 0.0748 \\
\hline APICAL_JUNCTION & 0.4436 & 0.5226 & 0.0930 & 0.5964 & 0.9420 \\
\hline APICAL_SURFACE & 0.2463 & 0.8867 & 0.0446 & 0.4599 & 0.7718 \\
\hline HEDGEHOG_SIGNALING & 0.6605 & 0.2274 & 0.3427 & 0.9753 & 0.3644 \\
\hline COMPLEMENT & 0.5503 & 0.2708 & 0.2752 & 0.7472 & 0.4735 \\
\hline UNFOLDED_PROTEIN_RESPONSE & 0.7689 & 0.7729 & 0.4700 & 0.0802 & 0.0279 \\
\hline
\end{tabular}




\begin{tabular}{|c|c|c|c|c|c|}
\hline & $P$-value & & & & \\
\hline PI3K_AKT_MTOR_SIGNALING & 0.0528 & 0.9677 & 0.6725 & 0.1803 & 0.3218 \\
\hline MTORC1_SIGNALING & 0.6152 & 0.9032 & 0.7798 & 0.8760 & 0.6822 \\
\hline E2F_TARGETS & 0.7822 & 0.7580 & 0.3937 & 0.0602 & 0.0556 \\
\hline MYC_TARGETS_V1 & 0.7708 & 0.6872 & 0.3228 & 0.0061 & 0.0012 \\
\hline MYC_TARGETS_V2 & 0.5094 & 0.3167 & 0.7014 & 0.1417 & 0.0170 \\
\hline EPITHELIAL_MESENCHYMAL_TRANSITION & 0.6721 & 0.9057 & 0.1794 & 0.7337 & 0.9523 \\
\hline INFLAMMATORY_RESPONSE & 0.7670 & 0.0732 & 0.1759 & 0.3939 & 0.8782 \\
\hline XENOBIOTIC_METABOLISM & 0.0106 & 0.5601 & 0.2595 & 0.2686 & 0.3029 \\
\hline FATTY_ACID_METABOLISM & 0.0679 & 0.6738 & 0.0601 & 0.0425 & 0.2397 \\
\hline OXIDATIVE_PHOSPHORYLATION & 0.4047 & 0.4305 & 0.0706 & 0.0045 & 0.0024 \\
\hline GLYCOLYSIS & 0.9342 & 0.6879 & 0.8739 & 0.7878 & 0.6407 \\
\hline REACTIVE_OXIGEN_SPECIES_PATHWAY & 0.3697 & 0.0708 & 0.6485 & 0.8970 & 0.4403 \\
\hline P53_PATHWAY & 0.1335 & 0.5546 & 0.5292 & 0.7423 & 0.8661 \\
\hline UV_RESPONSE_UP & 0.9160 & 0.0933 & 0.1971 & 0.7852 & 0.1948 \\
\hline UV_RESPONSE_DN & 0.0171 & 0.2320 & 0.6556 & 0.9801 & 0.3000 \\
\hline ANGIOGENESIS & 0.0793 & 0.2139 & 0.8252 & 0.0624 & 0.1576 \\
\hline HEME_METABOLISM & 0.5908 & 0.8810 & 0.8961 & 0.5243 & 0.5731 \\
\hline COAGULATION & 0.3290 & 0.4877 & 0.2534 & 0.5806 & 0.4252 \\
\hline IL2_STAT5_SIGNALING & 0.7320 & 0.0101 & 0.2977 & 0.9607 & 0.4088 \\
\hline BILE_ACID_METABOLISM & 0.9514 & 0.8629 & 0.1779 & 0.5036 & 0.6687 \\
\hline PEROXISOME & 0.7317 & 0.8256 & 0.3707 & 0.5001 & 0.1938 \\
\hline ALLOGRAFT_REJECTION & 0.5476 & 0.4178 & 0.6759 & 0.2342 & 0.1006 \\
\hline SPERMATOGENESIS & 0.0302 & 0.0799 & 0.9818 & 0.7270 & 0.3343 \\
\hline KRAS_SIGNALING_UP & 0.1073 & 0.8899 & 0.1848 & 0.0147 & 0.0314 \\
\hline KRAS_SIGNALING_DN & 0.3135 & 0.7944 & 0.6270 & 0.0895 & 0.1662 \\
\hline PANCREAS_BETA_CELLS & 0.1520 & 0.6471 & 0.9650 & 0.0140 & 0.0118 \\
\hline \multicolumn{6}{|l|}{ NS, non-TCM syndrome } \\
\hline
\end{tabular}




\section{Inflammation/immune cell type distribution among patients with or without Shi-Re diagnosis}

Our results showed a clear separation of patients with or without Shi-Re diagnosis based on the heatmap, and TGF $\beta$ and KRAS signaling activation were also identified as the hallmarks of cancer with Shi-Re diagnosis. As TGF $\beta$ and KRAS signaling activation contribute to shaping an inflammatory/immunosuppressive tumor microenvironment, we next evaluated the inflammation/immune cell type distribution in patients with or without Shi-Re diagnosis. Cell-type enrichment analysis of genes was performed using xCell. As shown in Fig. 3a, the heatmap revealed a varied inflammation/immune cell type distribution among patients with or without Shi-Re diagnosis. Compared with patients without Shi-Re diagnosis, patients with Shi-Re diagnosis showed significantly enriched mast cells, platelets and Tregs, but basophils, CLP, DC, and CDC cells were decreased (Fig. 3b). Therefore, these results suggested that Shi-Re ZHENG was associated with an inflammation/immunesuppressive tumor microenvironment in PDAC.

\section{Discussion}

In this study, we have for the first time performed plasma EV long RNA-seq to study the EV mRNA profiles in PDAC patients with different TCM ZHENG diagnoses. We observed a varied EV mRNA expression profile among patients with different ZHENG diagnoses. We also identified the hallmarks of cancer with different ZHENG diagnoses. In particular, we identified TGF- $\beta$ and KRAS signaling activation as the hallmarks of cancer with Shi-Re diagnosis, which may contribute to shaping an inflammation/immunesuppressive tumor microenvironment in PDAC.

EVs, a novel liquid biopsy, have been recognized as a valuable resource for cancer detection and monitoring [23-25]. The utility of EVs is particularly promising because of their presence in all biological fluids; in addition, EVs are secreted by all cells and their contents are stable and reliable and their potential multicomponent analyses. EV contents and amounts reflect the physiopathological state of the cells from which they are secreted. Depending on the cell of origin, EVs can contain many constituents, including DNA, RNA, lipids, metabolites, and cytosolic and cell-surface proteins [26]. Examination of EVs thus allows for a multicomponent diagnostic/prognostic window into disease detection and monitoring. The characteristic properties of EVs in delivering functional cargos to diseased cells also favor their use as therapeutic vehicles, both at the basic and applied levels [27]. In this study, we obtained plasma EV mRNA profiles in patients with different ZHENG diagnoses. We found that patients with different ZHENG diagnoses exhibited different EV mRNA profiles, which suggests that plasma EV mRNA detection has the potential to help differentiate TCM ZHENG.

The tumor microenvironment, especially the inflammatory and immune-suppressive microenvironment of pancreatic cancer, has a complex role in oncogenesis, tumor growth and therapeutic response. Pancreatic cancer can trigger an intrinsic inflammatory response that establishes a pro-tumorigenic microenvironment, which leads to remodeling of the tumor microenvironment through recruitment of 
leukocytes and lymphocytes, expression of tumor-promoting chemokines and cytokines, and induction of an angiogenic switch [28-30]. The production of growth factors and cytokines is one mechanism by which inflammation enhances pancreatic cancer initiation. Through activation of various downstream effectors, such as NF-KB, AP-1, STAT and SMAD transcription factors, cytokines control the immune and inflammatory milieu to either favor anti-tumor immunity (IL-12, TRAIL, IFNY) or enhance tumor progression (IL-6, IL-17, IL-23) and also directly affect cancer cell growth and survival (TRAIL, FasL, TNFa, EGFR ligands, TGF- $\beta$, IL-6) [31]. In this study, EV mRNA sequencing and subsequent pathway analysis revealed that many inflammatory and/or immune-suppressive pathways were enriched in patients with Shi-Re diagnosis, such as platelet degranulation, cellular response to interferon-gamma, fibrinolysis, and cellular response to interleukin-1. Therefore, our study suggests that the tumor microenvironment varies among patients with different TCM ZHENG.

In our study, typical gene sets associating with inflammatory and immune-suppressive microenvironment were enriched in the 50 hallmarks; for example, TGF- $\beta$ and KRAS signaling activation were enriched in patients with Shi-Re diagnosis. TGF- $\beta$ is an anti-inflammatory cytokine produced by cancer cells, myeloid cells, and T lymphocytes. TGF- $\beta$ signaling is an important regulator of the epithelial-mesenchymal transition and metastasis, and elevated TGF- $\beta$ is often associated with poor prognosis. TGF- $\beta$ activates SMAD transcription factors and MAPKs, which control expression of other regulators of the epithelialmesenchymal transition, such as Slug. However, TGF- $\beta$ also suppresses epithelial cell proliferation and early tumor growth, causing some tumors to acquire inactivating mutations in TGF $\beta$ signaling components [32]. Disruption of TGF $\beta$ signaling in cancer cells also results in upregulation of the SDF1 (CXCL12)-CXCR4 and CXCL5-CXCR2 chemokine: chemokine receptor pairs and induces rapid recruitment of MDSCs that promote metastasis and dampen the anti-tumor immune response [33]. The KRAS gene, as the most frequently mutated gene in PDAC, encodes a small GTP enzyme that mediates downstream signal transduction of growth factor receptors. KRAS plays widespread and essential roles in regulating cell growth, proliferation, differentiation, apoptosis, and other biological processes [34, 35]. Continuous activation of KRAS leads to aberrant signal transduction, uncontrolled cell proliferation, and inhibited apoptosis. As a result, activated KRAS may lead to tumor initiation [36]. KRAS has a marked effect in influencing the inflammatory and immune tumor environment. The influence of KRAS in antitumor immune response can be extensively affected by the infiltration of T cells and myeloid-derived suppressive cells (MDSCs) [37, 38]. KRAS-mutated cancer cells can exert on all kinds of immune cells by paracrine ways [39]. For instance, a high level of KRAS activity can produce many factors that maintain the inflammatory/ immune-suppressive microenvironment $[40,41]$. Our results suggest that patients with Shi-Re diagnosis exhibited an inflammation/immune-suppressive microenvironment, which may contribute to the progression of PDAC.

PDAC is also restriction of immune surveillance that supports tumorigenesis through paracrine cross-talk between tumor cells and immune cells. The tumor microenvironment contains innate immune cells (including macrophages, neutrophils, mast cells, myeloid derived suppressor cells, dendritic cells, and natural killer cells) and adaptive immune cells ( $T$ and $B$ lymphocytes) [42]. Therefore, we also evaluated the immune cell type distribution in patients with or without Shi-Re diagnosis. Mast cells, platelets and 
Tregs were significantly enriched but basophils, CLPs, DCs, and CDC cells were decreased in patients with Shi-Re diagnosis compared with patients without Shi-Re diagnosis. These results support our statement that Shi-Re ZHENG was associated with an inflammation/immune-suppressive tumor microenvironment in PDAC.

\section{Conclusions}

In summary, in this study, we found a varied EV mRNA expression profile among PDAC patients with different ZHENG diagnoses. We identified the hallmarks of cancer in patients with different ZHENG diagnoses. We identified TGF- $\beta$ and KRAS signaling activation as the hallmarks of Shi-Re ZHENG, which shape an inflammation/immune-suppressive tumor microenvironment in PDAC.

\section{Abbreviations}

EVs: Extracellular vesicles; PDAC: pancreatic ductal adenocarcinoma; TCM:

Traditional Chinese Medicine; TEM: transmission electron microscopy; SSC: side scattering; PBS: phosphate-buffered saline; PBMCs: peripheral blood mononuclear cells; SDS: sodium dodecyl sulfate; TPM: per kilobase million; GO: Gene Ontology.

\section{Declarations}

\section{Ethics approval and consent to participate}

The study was approved by the Fudan University Shanghai Cancer Center Ethics Committee (Ethical No.: 050432-4-1212B). The methods used in this study were performed according to the ethical standards of our committee and the tenants of the 1964 Declaration of Helsinki and its later amendments. Written informed consent was obtained from each patient according to the committee regulations.

\section{Consent for publication}

Not applicable.

\section{Availability of data and materials}

The data (figures and tables) and materials used to support the results of this study can be obtained from the first author upon request.

\section{Competing interests}

The author(s) declared no potential conflicts of interest with respect to the research, authorship, and/or publication of this article.

\section{Funding}


This study was supported by the National Natural Science Foundation of China (82025035, 8187198981622049); the Shanghai Science and Technology Committee Program (19XD1420900) and Shanghai Education Commission Program (17SG04).

\section{Authors' contributions}

SLY carried out the data collection and wrote the main manuscript text. YWG participated in the study design, analysis of data and acquisition of data. LQ, KC and YLZ participated in the data collection and preparation of the manuscript. SLH participated in the analysis and interpretation of data. YL and PW carried out the study design and analysis of data. All authors read and approved the final manuscript.

\section{Acknowledgments}

We thank Novel Bioinformatics Ltd., Co for the support in bioinformatics analysis with the NovelBio Cloud Analysis Platform.

\section{References}

1. Lu Y, Qi Y, Yan YH, Yao DN, Deng H, Deng JW,et al.Analysis of microRNA expression in peripheral blood moncytes of three Traditional Chinese Medicine (TCM) syndrome types in peoriasis patients. Chin Med. 2020冈doi囚1186/s13020-020-00308-y.

2. Wang FJ, Wang P, Chen LY, Geng YW, Chen H, Meng ZQ, et al. TAM infiltration differences in tumorfirst and ZHENG-first models and the underlying inflammatory molecular mechanism in pancreatic cancer. Integr Cancer Ther. 2018;17(3): 707-16.

3. Bao H, Gao J, Huang T, Zhou ZM®Zhang B®Xia YF. Relationship between traditional Chinese medicine syndrome differentiation and imaging characterization to the radio- sensitivity of nasopharyngeal carcinoma. Chin J Cancer.2010;11:937-45.

4. Lu C, Xiao C, Chen G, Jiang M, Zha QL, Yan XP, et al. Cold and heat pattern of rheumatoid arthritis in traditional Chinese medicine: Distinct molecular signatures indentified by microarray expression profiles in CD4-positive T cell. Rheumatol Int. 2012;32(1): 61-8.

5. Zhao HH, Chen JX, Shi Q, Ma XL, Yang Y, Luo LT, et al. Metabolomics-based study of clinical and animal plasma samples in coronary heart disease with blood stasis syndrome. Evid Based Complement Alternat Med. 2012; 2012:638723.

6. Dai JY, Sun SJ, Cao JM, Zhao Y, Cao HJ, Zheng NN, et al.Similar connotation in chronic hepatitis B and nonalcoholic Fatty liver patients with dampness-heat syndrome. Evid Based Complement Alternat Med. 2013; 2013:793820.

7. Song YN, Zhang H, Guan Y, Peng JH囚Lu YY, Hu YY, et al. Classification of traditional Chinese medicine syndromes in patients with chronic hepatitis B by SELDI-based proteinhhip analysis. Evid Based Complement Alternat Med. 2012; 2012:626320. 
8. Lu YY, Chen QL, Guan Y, Guo ZZ, Zhang HखZhang W\et al. Study of ZHENG differentiation in hepatitis B-caused cirrhosis a transcriptional profiling analysis. BMC Complement Altern Med.2014; 14:371.

9. Sun SS, Dai JY, Fang JW, Gou XJ, Cao HJ, Zheng NN, et al. Differences of excess and deficiency zheng in patients with chronic hepatitis B by urinary metabonomics. Evid Based Complement Alternat Med. 2013;2013: 738245.

10. Li XH, Jia LL, Liu XX. Preliminary discussion of diagnosis and treatment mode of future medicine from treating based on syndrome differentiation and precision medicine. Chin J Tradit Chin Med Pharm. 2018;33(11): 4789-92,

11. Hopkins Network pharmacology. Nat Biotechnol. 2007;25(10):1110-1.

12. Li S, Zhang B. Traditional Chinese medicine network pharmacology: theory, methodology and application. Chin J Nat Med. 2013;11(2):110-20.

13. Raposo G, Stoorvogel W. Extracellular vesicles: exosomes, microvesicles, and friends. J Cell Biol. 2013;200(4):373-83.

14. Thery C, Zitvogel L, Amigorena S. Exosomes: composition, biogenesis and function. Nat Rev Immunol. 2002;2(8):569-79.

15. Mc Namee N, O’Driscoll Extracellular vesicles and anti-cancer drug resistance. Biochim Biophys Acta Rev Cancer. 2018;1870(2) :123-36.

16. Shao H, Im H, Castro CM, Breakefield X, Weissleder R, Lee H. New technologies for analysis of extracellular vesicles. Chem Rev.2018; 118(4):1917-50.

17. EL Andaloussi S, Mager I, Breakefield XO, Wood MJ. Extracellular vesicles:biology and emerging therapeutic opportunities. Nat Rev Drug Discov. 2013;12(5):347-57.

18. Kalluri R, LeBleu The biology,function, and biomedical applications of exosomes. Science. 2020;367(6478): eaau6977,

19. Li SL, Li YC, Chen B, Zhao JJ, Yu SL, Tang Y, et al. exoRBase: a database of circRNA, IncRNA and mRNA in human blood exosomes. Nucleic Acids Res. 2018; 46 (D1): D106-12.

20. Li YC, Zhao JJ, Yu SL, Wang Z, He XG, Su YH, et al. Extracellular Vesicles Long RNA Sequencing Reveals Abundant mRNA, circRNA, and IncRNA in Human Blood as Potential Biomarkers for Cancer Diagnosis. Clin Chem. 2019;65(6): 798-808,

21. Yu SL, Li YC, Liao Z, Wang Z, Wang Z, Li Y, et al. Plasma extracellular vesicle long RNA profiling identifies a diagnostic signature for the detection of pancreatic ductal adenocarcinoma. Gut, 2020;69(3):540-50.

22. Hu XO, Wei B, Song YN, Ji Q, Li Q, Luo YQ, et al. Plasma metabolic profiling on postoperative colorectal cancer patients with different traditional chinese medicine syndromes. Complement Ther Med. 2018; 36:14-9.

23. Kalluri R. The biology and function of exosomes in cancer. J Clin Invest. 2016; 126(4):1208-15.

24. Kahlert $\mathrm{C}$, Kalluri Exosomes in tumor microenvironment influence cancer progression and metastasis. J Mol Med (Berl). 2013; 91(4):431-7. 
25. Bebelman MP, Smit MJ, Pegtel DM, Baglio SR. Biogenesis and function of extracellular vesicles in cancer. Pharmacol Ther. 2018; 188:1-11.

26. Shurtleff MJ, Temoche-Diaz MM, Schekman R. Extracellular vesicles and cancer: Caveat lector. Annu Rev Cancer Bio 2018; 2:395-411.

27. Van Niel G, D’Angelo G, Raposo G. Shedding light on the cell biology of extracellular vesicles. Nat Rev Mol Cell Biol. 2018;19(4): 213-28.

28. Mantovani A, Allavena P, Sica A, Balkwill F. Cancer-related inflammation. 2008;454(7203):436-44.

29. Soucek L, Lawlor ER, Soto D, Shchors K, Swigart LB, Evan GI. Mast cells are required for angiogenesis and macroscopic expansion of Myc-induced pancreatic islet tumors. Nat Med. 2007;13(10):1211-8.

30. Sparmann A, Bar-Sagi D. Ras-induced interleukin-8 expression plays a critical role in tumor growth and angiogenesis. Cancer Cell. 2004; 6(5):447-58.

31. Lin WW, Karin M. A cytokine-mediated link between innate immunity, inflammation, and cancer. J Clin Invest. 2007;117(5):1175-83.

32. Yang J, Weinberg RA. Epithelial-mesenchymal transition: at the crossroads of development and tumor metastasis. Dev Cell. 2008;14(6): 818-29.

33. Yang L, Huang JH, Ren XB, Gorska AE, Chytil A, Aakre M, et al. Abrogation of TGF beta signaling in mammary carcinomas recruits $\mathrm{Gr}-1+\mathrm{CD} 11 \mathrm{~b}+$ myeloid cells that promote metastasis. Cancer Cell. 2008;13(1):23-35.

34. Wood LD, Hruban RH. Pathology and molecular genetics of pancreatic neoplasms. Cancer J. 2012;18(6):492-501.

35. Tsai FD, Lopes MS, Zhou M, Court H, Ponce O, Fiordalisi JJ, et al. K-Ras4A splice variant is widely expressed in cancer and uses a hybrid membrane-targeting motif. Pro Natl Acad Sci U S A. 2015;112(3):779-84.

36. Spaargaren M. Bischoff JR, McCormick Signal transduction by Ras-like GTPases: a potential target for anticancer drugs. Gene Expr. 1995;4(6):345-56.

37. Pylayeva-Gupta Y, Grabocka E, Bar-Sagi D. RAS oncogenes: weaving a tumorigenic web. Nat Rev Cancer. 2011;11(11):761-74.

38. Steele CW, Jamieson NB, Evans TR, Mckay CJ, Sansom OJ, Morton JP, et al. Exploiting inflammation for therapeutic gain in pancreatic cancer. Br J Cancer. 2013;108(5): 997-1003.

39. Dias CP, Guimaraes CF, Cardoso AP, Mendonca S, Costa AM, Oliveira MJ, et al. KRAS oncogenic signaling extends beyond cancer cells to orchestrate the microenvironment. Cancer Res. 2018;78(1):7-14.

40. Charo C, Holla V, Arumugam T, Hwang R, Yang PY, Dubois RN, et al. Prostaglandin E2 regulates pancreatic stellate cell activity via the EP4 receptor. Pancreas. 2013;42(3): 467-74.

41. Cheng H, Fan K, Luo GP, Fan ZY, Yang Chao, Huang QY, et al. KrasG12D mutation contributes to regulatory $T$ cell conversion through activation of the MEK/ERK pathway in pancreatic cancer. Cancer Lett. 2019; 446:103-11. 
42. de Visser KE, Eichten A, Coussens LM. Paradoxical roles of the immune system during cancer development. Nat Rev Cancer. 2006; 6(1):24-37.

\section{Figures}

a

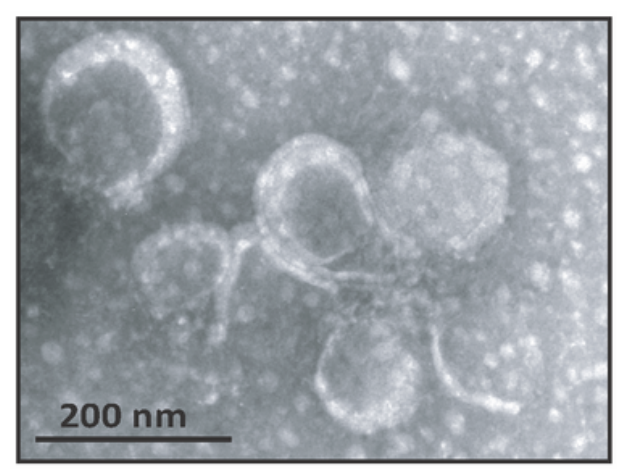

C

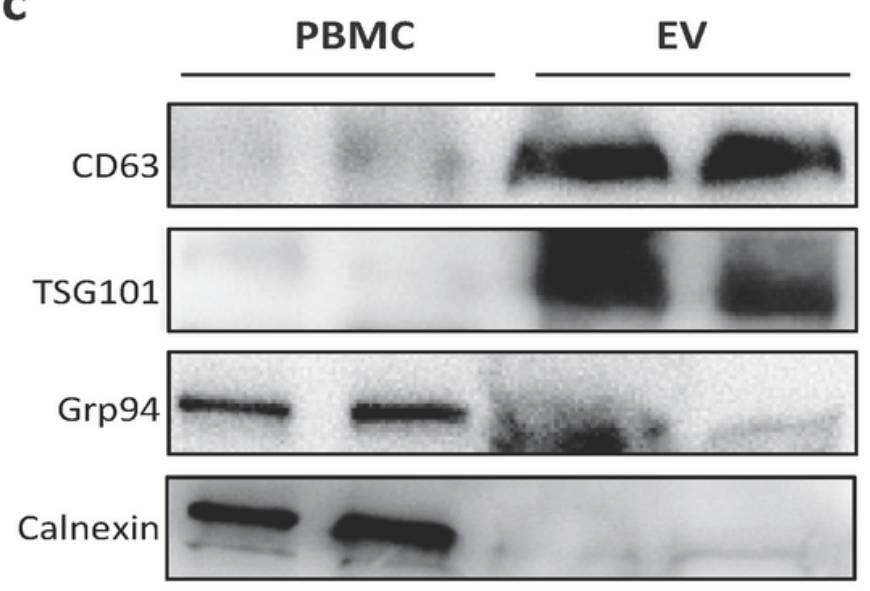

b

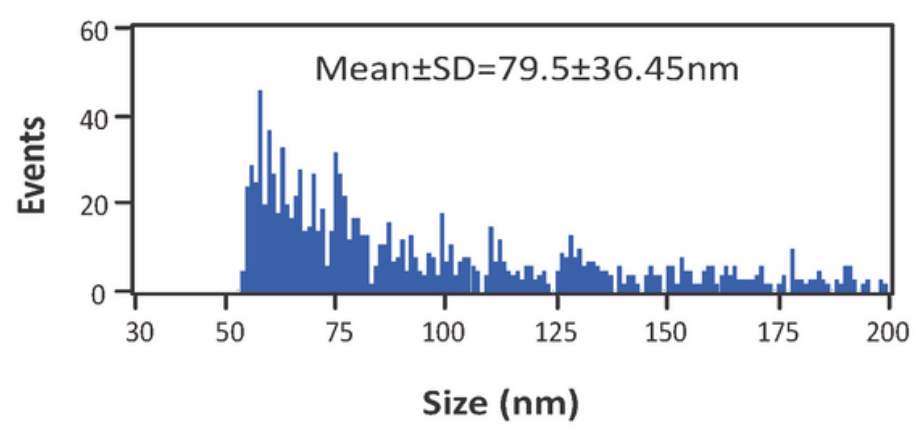

d

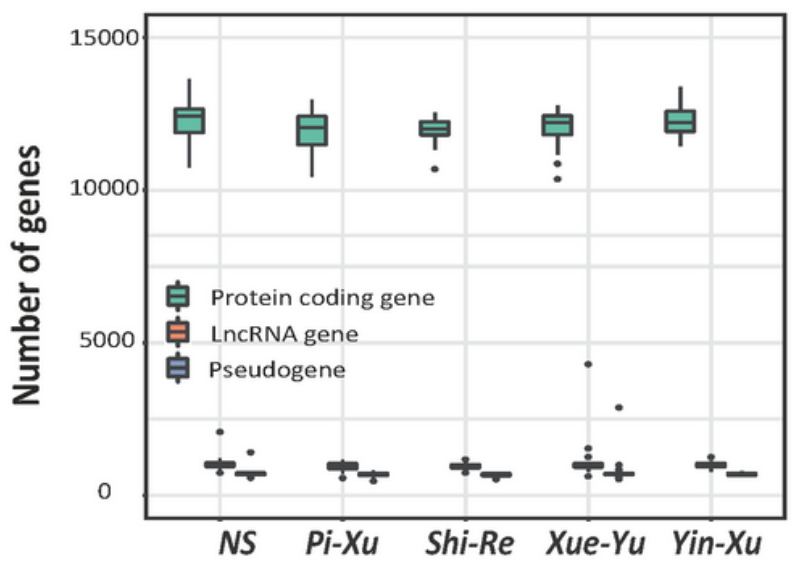

\section{Figure 1}

Verification of plasma EV and long RNA sequencing ( $a-b)$ EVs were detected by transmission electron microscopy (a) and flow cytometry (b). Scale bar, $200 \mathrm{~nm}$. (c) Western blots of EV markers TSG101 and TCD63 in isolated vesicles. Grp94 and calnexin, observed in PBMCs but not in the isolated vesicles, were used as controls. (d) Distribution of EV long RNA per sample among patients with different ZHENG diagnoses. 
a
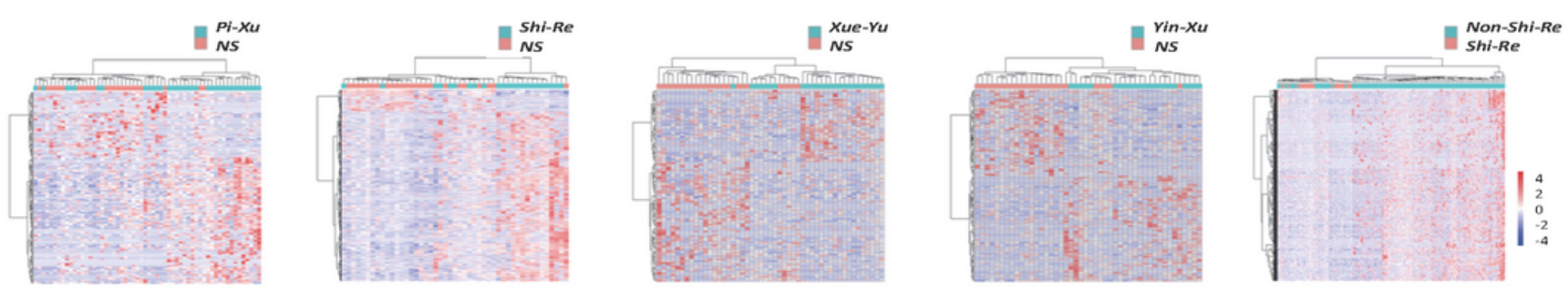

b

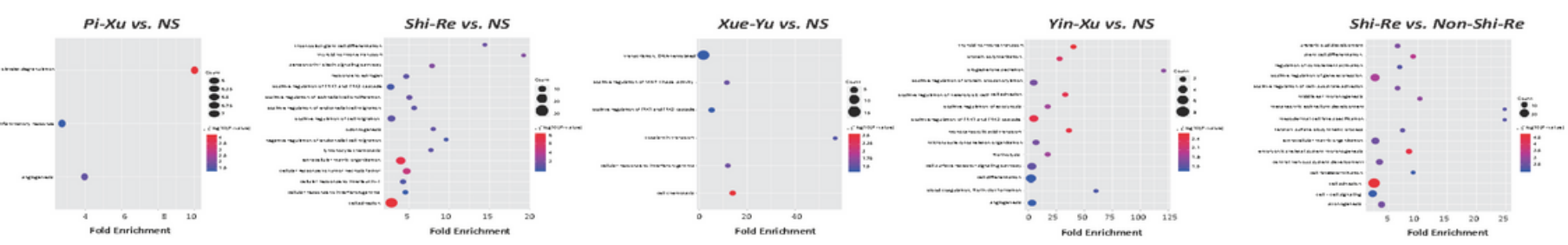

Figure 2

Hierarchical clustering and pathway enrichment analysis of EV mRNAs among patients with different ZHENG diagnoses. (a) Unsupervised hierarchical clustering of differentially expressed EV mRNAs in patients with different ZHENG diagnoses. (b) GO pathway enrichment analysis.

a

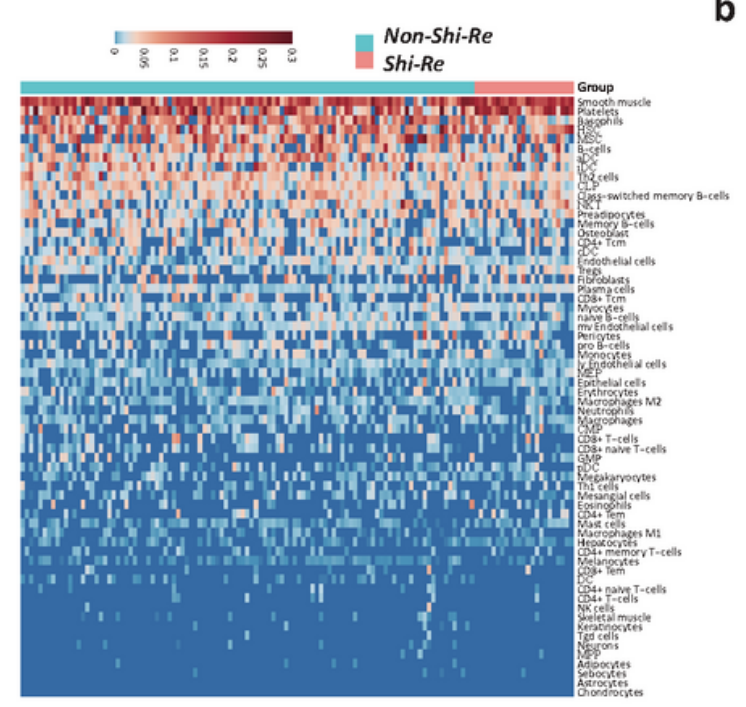

b

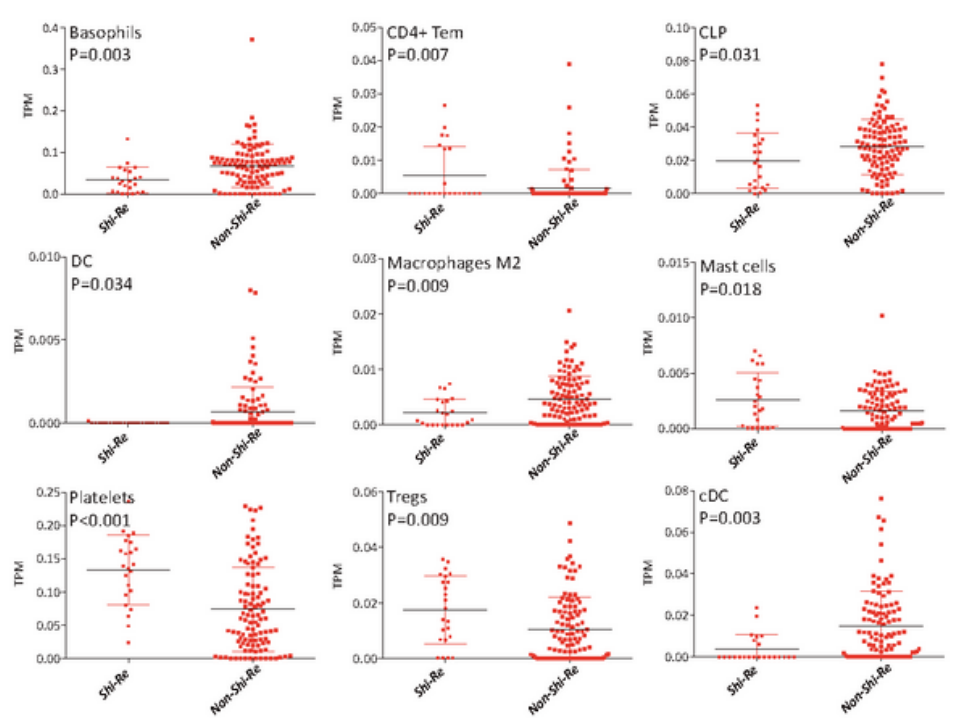

\section{Figure 3}

Cell-type distribution among patients with or without Shi-Re diagnosis (a) The heatmap of the cell-type distribution between patients with or without Shi-Re diagnosis. (b) The scatter plot of the cell-type in patients with or without Shi-Re diagnosis. 